\title{
Dynamic Behaviour Analysis of a Dual-Rotor System Using the Transfer Matrix Method
}

\author{
B.B. Maharathi \\ Department of Mechanical Engineering, IGIT, Sarang - 759146, Orisa, India
}

\section{P.R. Dash}

Department of Mechanical Engineering, UCE, Burla, Orissa, India

\author{
A.K. Behera ${ }^{\dagger}$ \\ Department of Mechanical Engineering, NIT, Rourkela, Orissa, India
}

(Received 28 February 2001; accepted 16 April 2004)

\begin{abstract}
This paper presents a general formulation for the problem of the steady-state unbalance response of a dual rotor system with a flexible intershaft bearing using an 'extended' transfer matrix method, where the transfer matrix assumes a dimension of $(33 \times 33)$. The validity of the formulation is established by comparing the results obtained through a computer program with closed form solutions available for some simple cases. Some interesting phenomena of steady-state whirl orbits of the dual rotor system are described.
\end{abstract}

${ }^{\dagger}$ Member of the International Institute of Acoustics and Vibration (IIAV)

\section{Nomenclature}

$[A],[B]\}-$ Represent transfer matrices,

$[C],[D]\}^{-}$each of dimension $(33 \times 33)$

$[\bar{B}] \quad-$ Bearing matrix

$C_{y y}, C_{z z}$ - Direct damping coefficients

$C_{y z}, C_{z y}$ - Cross coupled damping coefficients

$C_{H}, C_{L}-$ High and low rotor damping, respectively

E - Modulus of elasticity

e $\quad-$ Eccentricity

$[F] \quad-$ Field matrix

$[\bar{F}] \quad-$ Overall field matrix

$h \quad-$ Thickness of the disk

[I] - Unit matrix

$I_{T} \quad-$ Transverse mass moment of inertia of the disk

$I_{P} \quad-$ Polar mass moment of inertia of the disk

$K_{y y}, K_{z z}$ - Direct stiffnesses

$K_{y z}, K_{z y}-$ Cross coupled stiffnesses

$K_{H}, K_{L}-$ High and low rotor stiffness, respectively

$l \quad$ - Sectional length

$M_{y}, M_{z}$ - Moments about $y$ - and $z$-axes, respectively

$M_{H}, M_{L}-$ High and low rotor mass, respectively

$m \quad-$ Mass of the element per unit length

$m_{b} \quad$ - Mass of the shaft at bearing station

$m_{e} \quad$ - Unbalance mass

$[P] \quad$ - Point matrix

$[\bar{P}] \quad-$ Overall point matrix

$U_{y}, U_{z} \quad$ - Unbalance components

$V_{y}, V_{z}$ - Shear force in $y$ - and $z$-directions, respectively

$v, w \quad$ - Deflection along $y$ - and $z$-axes, respectively

$X, Y, Z \quad$ - Fixed co-ordinate system

$\bar{X}, \bar{Y}, \bar{Z}$ - Rotating co-ordinate system
$\{\bar{S}\} \quad$ - Modified State vector at any station

$\left.\{S\}_{O A},\{S\}_{O B}\right\} \quad$ Represent end conditions

$\left.\{S\}_{O C},\{Z\}_{O D}\right\}^{-}$of the dual rotor system

$\omega_{m}, \omega_{n}$ - Inner and outer rotor speeds, respectively

$\theta, \phi \quad-$ Slope in $x-z$ and $y-z$ planes, respectively

$\beta-$ Angular position of unbalance

$\lambda$ - Eigenvalue

\section{Subscripts}

$m, n \quad$ - Pertaining to the effect of inner and outer rotor speeds, respectively

c - Cosine component

cm,cn - Cosine components of inner and outer rotor, respectively

$s \quad-$ Sine component

sm, sn - Sine components of inner and outer rotor, respectively

$y \quad-$ Along $y$-axis

$z \quad-$ Along $z$-axis

$j \quad-\quad$ Pertaining to a junction

\section{Superscripts}

$R \quad$ - Right to a section

$L \quad$ - Left to a section

$t \quad-$ Transpose of a matrix

\section{INTRODUCTION}

With the ever increasing demand of large power and smaller gas turbine engines for aircraft propulsion, a two spool system with intershaft bearings is becoming a standard layout to accommodate the compressor and turbine rotors. 